\title{
artículos
}

\section{Un histórico complejo arquitectónico y paisajístico de características homogéneas: las Villas Tuscolanas de la época moderna}

Rodolfo Maria Strollo

Università Roma Tre

PALABRAS CLAVE: Arquitectura contemporánea/ Italia

RESUMEN

El Complejo de las Villas Tuscolanas constituye un grupo de edificios monumentales - a menudo inscritos en grandes complejos externos - que pueden ser considerados globalmente como un vasto y articulado fenómeno arquitectónico y paisajístico, cuya particularidad ha suscitado gran interés en muy diversos ambientes y momentos, manifestandose en una determinada - pero no limitada - extensión territorial: la lade ra Tuscolana del relieve lacial de los Colli Albani. Se reconocen los siglos XVI y XVII como el período de máximo esplendor, aunque el fenómeno se fija en la época romana, e igualmente contemplan significativas transformaciones en tiempos recientes. Su vasta extensión territorial junto con los aspectos socio-económico-politicos, artístico-figurativos y geográfico-territoriales propios de su historia, muestra cuán amplia y complicada puede ser una lectura global del fenómeno.

\section{ABSTRACT}

The group of monumental handiworks - often comprehending some large external fittings - commonly pointed out as the Complex of Ville Tuscolane, may be regard$e d$ as a large and articulated architectural and landscape phenomenon which invested several ambits and many ages, inside a well determined area: the slope of the ancient Tusculum on the relief of the Alban Hills (in the Latium Region). The XVI and XVII Centuries are the period of the maximum shine, but the phenomenon itself has its roots in the Roman epoch and it has been passing through many meaningful transformations in the recent past. The considerably large territory with this feature, besides to many socio-economical, political, artistic, geographic and territorial, aspects inherent in its history, may well show how ample and complicated may result an attempt to give an "all-round" reading of the phenomenon itself.

Se define como Complejo de las Villas Tuscolanas el conjunto de obras monumentales edificadas durante el Renacimiento, en las inmediaciones de Roma, para ser la residencia temporal de las más importantes familias vinculadas por títulos diferentes a la Corte pontificia. El mayor interés de estas Villas está posiblemente en el hecho de que ellas, no tanto constituyen un conjunto de edificios individuales de más o menos alta cualidad arquitectónica, sino un grupo coherente, a pesar de la volun-

* STROLLO, R.: "Un histórico complejo arquitectónico y paisajístico de características homogéneas : las Villas Tuscolanas de las época moderna", Boletín de Arte, $\mathrm{n}^{\circ} 28$, Departamento de Historia del Arte, Universidad de Málaga, 2007, págs. 9-22. Traductor: Arnulfo Martínez Portales. 
tad de rivalizar que, a menudo, caracteriza a sus propietarios. Además de las características tipológicas y morfológicas de cada una de las obras construidas, relacionadas estrechamente a la orografía del lugar ${ }^{1}$, el Complejo, en efecto, alcanza cualificación debido a la presencia de recorridos arboleados, quintas vegetales, bosques y jardines, organizados de tal modo que se enlazan en un "todo único".

Las construcciones de este grupo - strictu sensu - fueron doce 2 ; sin embargo, no deben olvidarse los centros religiosos correlacionados directamente con éstas, y algunas otras podrían también considerarse, pues se encuentran en los alrededores y remarcan en parte las características, pero con un tono menor ${ }^{3}$; ni se puede soslayar el hecho que, de las construcciones mayores, solamente nueve se mantienen en pie (tres quedaron destruidas durante el último conflicto mundial) ${ }^{4}$ [1]

La historia de las Villas - que comprende construcciones principales y accesorias, y, además, disposiciones ambientales y paisajísticas complejas que, por lo

\footnotetext{
1 El relieve lacial de los Colli Albani, ubicado al sureste de la Capital, es de naturaleza orográfico-geológica volcánica (en efecto, se le denomina también Volcán Lacial). Situado en posición estratégica -en medio de

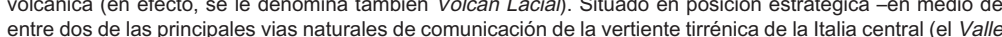
del río Sacco y la Llanura Pontina), mientras el tercer eje (llamado en la época clásica vía Latina) que lo atradel río Sacco y la Llanura Pontina), mientras el tercer eje (llamado en la época clásica vía Latina) que lo atra-
viesa justo por el medio - se caracteriza, asimismo, por sus benignas condiciones climáticas y geopedológicas. De ahí que, desde las épocas más remotas, existen, pues, documentos que informan sobre los asentamientos humanos, por lo general, de consideración. La vertiente del Tuscolano que se asoma a la llanura del Tiber, a la que se impalma con un curso altimétyrico no muy escarpado, está orientado hacia el septentrión lo cual no le impidehallarse óptimamente solendo y gozar de un clima templado, y, además, por estar a poca distanzia de la costa tirrénica. Con la aparición y crecimento del poder de Roma, empezó, pues, a desarrollarse aquí el fenómeno de las residencias temporales: surgieron múltiples Tusculani secessus (ricas moradas de verano de las familias patricias, donde podían gozar, en la quietud agreste y a escasa distancia de la das de ciudad, are sanes, también. Lo que deviene durante el Renacimiento representa, por muchos aspectos, la reaparición (a menudo, ideológicamente motivador reposo" y explotación agrícola de estas tierras (ésta se manifestó, sobre todo, en el cultivo de la vid y de olivo, que por largo tiempo se constituyeron en vocación privilegiada, dentro de dimensiones que, aunque no se debían pasar por alto, resultaban, sin embargo, muy limitadas al comparárselas.

2 A. Kircher en su Latium id est nova et parallela Latii tum veteritum novi descriptio, Amsterdam 1671 anota «...duodecim villae...» y C.B. Piazza en La gerarchia Cardinalizia, Roma 1703, también: «...de entre el resto de villas, doce son las más ilustres y conspicuas que a vista de respetables forasteros son un esp de villas, doce son las más ilustres y conspicuas que a vista de respetables forasteros son un espectáculo se les atribuyeron en el pasado en correspondencia con los múltiples pasajes de propiedad: Aldobrandini (Belvedere), Falconieri (Rufina - Ruffina), Grazioli (Acquaviva - Bracciano - Carafa - Montalto - Odescalchi), (Belvedere), Falconieri (Rufina - Ruffina), Grazioli (Acquaviva - Bracciano - Carafa - Montalto - Odescalchi),
Lancellotti (Bonanni - Piccolomini - Visconti), Mondragone, Muti (Arrigoni - Cesaroli - Rocci - Varesi), Lancellotti (Bonanni - Piccolomini - Visconti), Mondragone, Muti (Arrigoni - Cesaroli - Rocci - Varesi),
Pallavicini (Belpoggio - Ceri - Sciarra), Parisi (Borghese - Borghesiana - Taverna), Rufinella (Deti - Ferreria - Ruffinella - Sacchetti - Tuscolana), Sora (Boncompagni - Torricella), Torlonia (Altemps - Conti - Galli Ludovisi), Vecchia (Angelina - Tuscolana).

3 Otras construcciones diferentes y más o menos grandes que destacan podrían incluirse (a pleno título) en el citado grupo gracias a las recargadas semejanzas histórico-artístico-sociales a la ya mencionada docena "clásica". Nos referimos, por ejemplo, al Palazzetto Mergé, a Villa Cavalletti, a los Casini Pescatore en la zona de Frascati, pero también a la Villa di Campovecchio, en territorio de la cercana Grottaferrata. Todos estos casos, muchos textos no lo mencionan, quizás por las dimensiones más sobrias que las de las doce villas, casos, muchos textos no lo mencionan, quizás por las dimensiones más sobrias que las de las doce villas, mente; inclusive hasta en el nombre: en efecto, con la denominación Villas de Frascati se indicaba las Villas Tuscolanas (apelativo que hasta hoy viene usado, sobre todo por estudiosos que no son italianos).
} 


\section{Q E artículos Un histórico complejo arquitectónico y paisajístico...}

1. Localización de las villas.

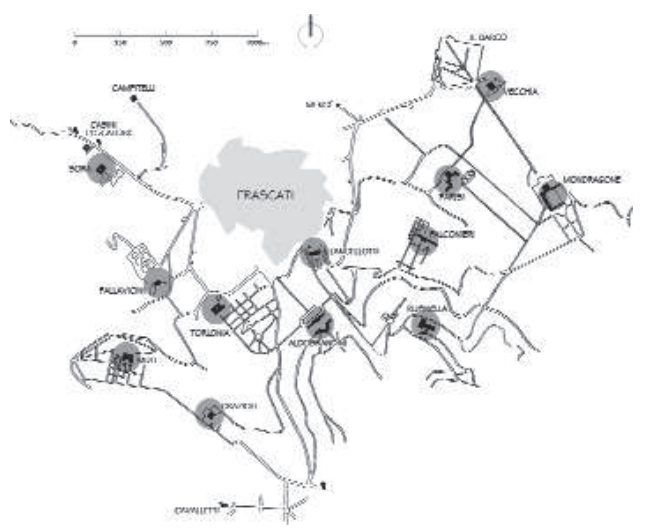

general, asumen importancia y valor semejante o hasta superior a las de las construcciones - puede esquematizarse en cuatro fases fundamentales: a las tres primeras hacen referencia la mayor parte de los autores que han tratado el tema; la cuarta la definen hoy los casos más recientes, ocurridos a partir de inicios del siglo pasado 5 .

- Las estructuras originarias de las Villas Tuscolanas surgieron, en su mayor parte, sobre los restos de las estructuras de la época romana, en el lapso de tiempo

4 Las villas Pallavicini, Torlonia y Vecchia fueron destruidas por los bombardeos de la segunda conflagración mundial.

5 La producción historiográfica referida al Complejo comprende, además de las numerosas contribuciones relativas a cada una de las Villas - un número menor de estudios que han tratado el tema con un alcance mayor, abrazando el articulado fenómeno que el sistema ha definido y estructurado como tal, o, poniéndo el acento en su caracterización como unicum homogéneo. La obra que destaca en este sentido es, por clerto la de FRANCK, C.: Die Barockvillen in Frascati, München-Berlín, 1956 (después The Villas of Frascati, London-New York 1966) aunque no trata sobre la Villa Sora. Otros textos han hecho un estudio "general" de las Villas, analizando una o más (a veces todas) de manera singular, sin profundizar la temática en sus aspectos "mancomunantes": entre estos basta citar a BELLI, I.; BRANCHETTI, M.G.: Ville della Campagna Romana, Milano 1975, y a COFFIN, D. R.: The Villa in the life of Renaissance Rome, Princeton, 1979. Elementos de interés emergen del ensayo de GUERRINI, P.: "La funzione delle ville nello sviluppo del paese", en TANTILLO, A.: Villa e Paese-Dimore nobili del Tuscolo e di Marino (catálogo de la exposición documental), Roma 1980, págs. 39-73) y del de TERRANOVA, A.: Paesaggio di villa: Trionfo, crisi, attualita dei modelli umanisti di annessione cultuale della natura nelle Ville Tuscolane, en PARIS, T:L ( Castelli Romani, Quaderni di documentazione per una storia urbanistica edilizia ed artistica della Region Lazio, Roma, 1981, págs. 315-366. Estos trabajos analizan, sin embargo, aspectos específicos de la cuestión y, además, los han integrado - como específicas contribuciones - en publicaciones cuyo interés total es de naturaleza diversa (en el primer caso, tratándose de una exposición organizada por la Superintendencia de Bienes Artísticos e Históricos de Roma la atención se dirigía, de modo especial, a los frescos de las Villas; en el segundo, los aspectos paisajísticos e históricos más generales de un área mucho más extensa). Un programa de tutela urbanística que consideraba el entero Complejo lo elaboró V. Ghio Calzolari, Una proposta per le Ville di Frascati, en BELLI BARSALI, I.: Per le Ville di Roma e del Lazio, catá- 


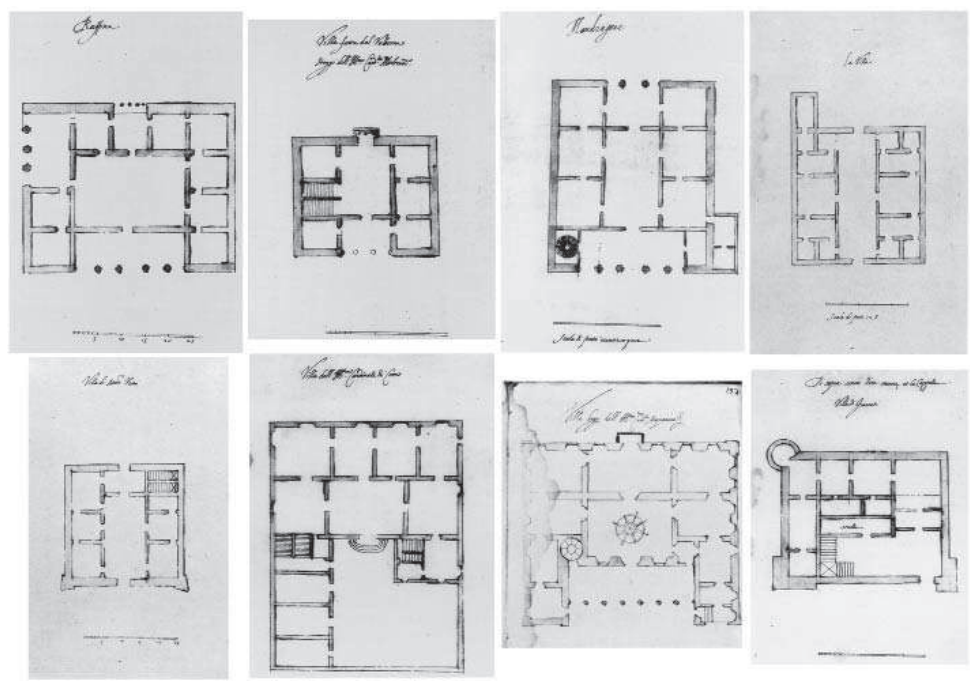

2. Villas Tuscolanas.

que coincide, aproximadamente, con la segunda mitad del siglo XVI (1548-1607). EI primer impulso de esta fase de desarrollo se identifica con la ejecución - por voluntad del Papa Paulo III (Alejandro Farnese, 1534-1549) - de la Rufina (hoy Falconieri), al sur de la ciudad, cuesta arriba respecto a ella, en una cima del relieve tuscolano 6 [2].

En 1537, Frascati (perteneciente a los Colonna y a los della Rovere en el cuarto de siglo anterior) había sido, efectivamente, "reconquistada" por la Rev.

logo de la exposición organizada por las sección romana de Italia Nostra, Roma, 1978, págs. 140-163. Tratados referidos a aspectos mancomunantes y de definición histórica del fenómeno se encuentran en STROLLO, R. M.: II Complesso delle Ville Tuscolane: considerazioni sulle fasi evolutive, en Id., Architettura e ambiente: casi di studio, Roma 2004, págs. 195-228, y en Id. Per lo studio di un fenomeno architettonico e paesistico storico circoscritto: le Ville Tuscolane, en las Actas del Congreso Internacional "Residenze, Ville e paesistico storico circoscritto: le Ville Tuscolane, en las Actas del Congreso Internacional "Residenze,

6 Cfr. ZIDEK,F.: Villa Falconieri, en "I. Jahresbericht des Gymnasium der Gesellschaft Jesu in Kalksburg", Wien 1907; PORTOGHESI,P. "L'opera del Borromini nel palazzo della Villa Falconieri", en Quadern dell'Istituto di Storia dell'Architettura, núm. 14, Roma 1956, págs. 7-20; DEVOTI, L.: Campagna Romana viva Le Ville Tuscolane. Villa Ruffina Falconieri, Frascati 1986; STROLLO, R. M.: "Villa Ruffina Falconieri, la villa 
Cámara Apostólica7. Paulo III, siguiendo los pasos del "Papa humanista" Pío II (Enea Silvio Piccolomini, 1458-1464) a quien se debe la inicial denominación urbana de Frascati ${ }^{8}$, la modernizó - conmemorándola como Tuscolo restituida ${ }^{9}$ - dando impulso, así, al fenómeno de las Villas, y orientando el desarrollo de la ciudad hacia los puntos de conexión con los diferentes lugares en los que ello tomaba cuerpo.

El patriciado romano del Renacimiento - representado por la más alta Jerarquía eclesiástica y por las familias que fundaban su poder en las finanzas - va "acuartelándose" en los Colli Tuscolani, con la firme voluntad de transcurrir su estadía según el criterio del otium romano - reconsiderado inclusive en clave de meditación espiritual, o, sencillamente, por el solo gusto de hacer competir la posesión de la estructura destinada a tales usos 10

Gracias a un extendido clima, socialmente más sereno y económicamente más florido; asimismo, al florecimiento cultural y al renovado interés por los clási-

Tuscolana "del Borromini"', en Id., Contributi sul Barocco Romano: rilievi, studi e documenti, Roma, 2001, págs. 133-173.

7 El 21 de agosto de 1511, el Papa Julio II (Giuliano della Rovere, 1503-1513) donó "in perpetuum" el Castillo de Frascati a los cónyuges Marcantonio Colonna y Lucrezia Franciotti della Rovere (además de acreedor, valiente capitán de las milicias pontificias, el primero; y, sobrina del Pontífice por parte de madre - Luchina della Rovere - la segunda). Lucrezia quedó viuda en 1522, y quince años más tarde cedió la propiedad a Pier Luigi Farnese, hijo de Paulo III, quien el 11 de abril de 1537 la canjeó por el Ducado de Castro de la Rev. Cámara Apostólica; cfr. A. Ilari, Documenti peer la storia di Frascati - III - i d'Estouteville ed i Colonnadella Rovere (1478-1537), en "Archivi", a. XXIII, fasc.2-3, 1956; Id., Frascati fra Medio Evo e Rinascimento con gli statuti esemplati nel 1515 e altri documenti, pág. 110, Roma 1965; G. y F. Tomassetti, La Campagna Romana antica, medievale e moderna, vol. IV, Via Latina, Roma 1926, reimpreso Sala Bolognese 1976 luego reeditado - con el nombre solo de Giuseppe - en una n. ed. agg. dirigida por L. Chiumenti, F. Bilancia, Città di Castello 1976 (Florencia 1979 ed. cons.), págs. 401-403.

8 Para conocer la historia urbanística de Frascati véase, además de A. Ilari, Frascati..., Op. Cit.; LANCIANI, R.: "La riedificazione di Frascati per opera di Paolo III", en el Archivio della Regia società romana di Storia patria, a. XVI, págs. 517-522, Roma 1983; ZOCCA, M.: "Sistemazioni urbanistiche del Rinascimento ne Lazio", en Palladio, a. VII, nº. 1, 1943, págs. 40-50; CASTAGNOLI, F.; CECCHELLI, G.; GIOVANNONI, G. et al., Topografia e urbanistica di Roma, Bolonia, 1958. GIOVANNONI, G.: Antonio da Sangallo il Giovane, Roma, 1959.

Esta locución - bajo la forma de TUSCOLO REST - aparece en la medalla cuya creación pertenece a Gian Federico Bonzagna (conocido también como Federico da Parma o Federico Parmese) que, con motivo de la edificación de la primera villa (la Rufina) y de la contemporánea reorganización de la ciudad, el Papa ordenó ex profesamente su elaboración. La medalla se conserva (junto a tres ejemplares similares) en las Civiche Raccolte Numismatiche de Milán, en bronce dorado, de diámetro $36 \mathrm{~mm}$; el Bollettino di Numismatica del Raccolte Numismatiche de Milan, en bronce dorado, de diametro $36 \mathrm{~mm}$; el Bollettino di Numismatica del
Ministero dei Beni Culturali e Ambientali la describe (Monografía 4.II.I, a. 1988 - Catálogo de las Medallas II. Siglo XVI, a. V, págs. 117-118, fol. 981-984). Una reproducción de ella aparece en F. Bonanni, Numismata Pontificum Romanorum Quae A Tempore Mertini v Usque ad Annum MDCXCIX Vel Autoritate Publica Vel Privato Genio In Lucem Prodiere, Roma, 1699, tabla xxvII.

10 En cuanto al comportamento competitivo que se gestó en las poderosas familias, hasta el punto de provocar fenómenos de ostentación, basta recordar la nota polémica que sostuvo otro célebre "veraneante" (el vocar fen cun "buente (e Cardenal Cesare Banio, 1538tuscolano, del loma MORITURO SATIS (de gan signicación para quien debe moin). Además, se puede captar otra manifestación de verdaderos lemas de invidia - que ni el Papado se salvaba - en las palabras del diarista vaticano, que Grossi Gondi lo señala, a propósito de la impetuosa ira que envuelve a Paulo V (Camillo Borghese, 1605-1621), apenas diose cuenta de la poca disponibilidad de agua en "su" Mondragone con respecto a la que gozaba en la Villa Belvedere «...sospechoso por haber visto aumentar el de 

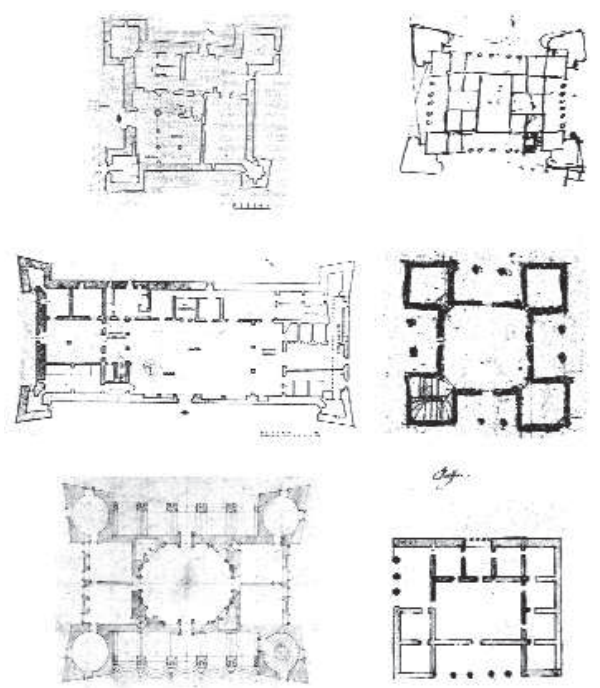

4

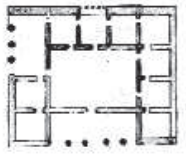

$-\cdots=$

3. Casini.

$\cos ^{11}$, se consiguió el redescubrimiento de estos lugares, y el empuje a la edificación se debió, una vez más, a las condiciones favorables del clima, seco y ventilado en verano (y más bien templado en inverno), a la fertilidad del terreno y a una relativa disponibilidad de recursos hídricos que, además de favorecer los cultivos productivos y la plantación de alamedas ornamentaales, permitió poco después embellecer los parques con fastuosos juegos de agua.

A tales dotes naturales, añadíanse la espléndida vista de Roma y de la Campagna Romana, y el fácil hallazgo (en la misma zona) de materiales de construc-

Aldobrandino, ha tratado de coger a ese arquitecto para hacerlo colgar, pero no lo ha logrado...» cfr. Grossi Gondi, Le Ville Tuscolane nell'epoca classica e dopo il Rinascimento. La Villa dei Quintilli e la Villa di Mondragone, Roma 1901, pág. $90, \mathrm{n}^{\circ}$. 2. Aunque sin llegar a tremenda ferocidad, encontramos anotaciones de verídicos actos de vandalismo en perjuicio de la propiedad adyacente, como el que encargó el marqués Paolo Sforza, propietario de la Villa Rufina a finales siglo XVl: " ha mandado a S. Torquato Conti de Valmontone con muchos hombres para que arruinen todas las fuentes y conductes de la propiedad de Valmontone con Cardenal [Ferreri, propietario de la Rufina], quien enterado de ello ha mostrado gran resentimiento ante S.
S.ta...»; Cfr. COFFIN, D.R.: The Villa in..., Op. Cit., pág. 52, $\mathrm{n}^{\circ} 130$.

11 El caso del célebre humanista y literato Annibal Caro es, en este sentido emblemático, pues compró una propiedad en Frascati que la eligió como lugar de espera, entre 1563 y 1566, año de su muerte, de la traducción al italiano de la Eneida de Virgilio. 
ción de apreciables calidades: la Pietra Sperone del Tuscolo, el Peperino, el Basalto y la Pozzolana, y, además, de madera (a vasta disposición gracias a las extensas áreas boscosas que los circundaban) ${ }^{12}$.

A diferencia de otras realidades análogas (por ejemplo, las Villas Venetas) en las unidades del Complejo Tuscolano no se contempló de forma sistemática ni mucho menos en tono representativo, el componente "agrícolo-productivo" de los estratos del terreno. Lo demuestran - además de las actitudes "culturales" de los propietarios - el tipo de cultivos prevalentes (poco laboriosos, como el del olivo, por ejemplo), la ausencia de edificaciones de servicio (almacenes, graneros, depósitos, etc.), y otras caracteríscas más, propias de un grupo unido y "arrocado" en las boscosas laderas del Colle del Tuscolo, carente por ello de tales extensos terrenos que podian justificar estructuras de doble funcionalidad.

La base planimétrica de estos primeros núcleos, llamados Casini (casas de campo que, por sus dimensiones, no eran nada modestas) se conecta a la tradición toscana de la villa-castillo, en la que por entre las torres angulares que una vez más traen la memoria medieval de la construcción fortificada, se insertan las espaciosas galerías que los tratadistas del Renacimiento, a menudo, describen, icone de la nueva aspiración humanista al goce del paisaje y de la naturaleza. Semejantes signos precursores pueden atribuirse, también, a la actividad in situ de un círculo de artistas toscanos de confianza de los Farnese ${ }^{13}[3]$.

Si todos los esquemas planimétricos se referían a un amplio espacio central en torno al cual se distribuian las habitaciones más pequeñas y los ambientes porticados, en los alzados las obras se caracterizaron por un contrapunto de vistas panorámicas que entraba en juego en dos direcciones opuestas: el monte (al sur), en el que se divisaba el boscoso relieve del Tuscolo; el valle (al norte), permitía divisar la ciudad de Roma, cuyo lecho se extendía desde el monte Soratte hasta el mar Tirreno.

Las diferencias entre las unidades que constituyeron el Complejo debiéronse a sutilezas en la solución de las aberturas (las exigencias de orientación las determinaron) y a las características de los lugares utilizados que, en el breve periodo de

12 Cfr. BALDONI, C.;STROLLO, R. M.: "I materiali delle Ville Tuscolane", en Castelli Romani, a. XL (VIII n. s.), nº. 2, mar. - apr. 2000, págs. 53-63.

13 Además de Antonio da Sangallo il Giovane, Bartolomeo Baronino es otro técnico indicado como operador en aquel periodo en Frascati (Cfr. LANCIANI, R.: La riedificazione ..., Op. Cit. e Ídem., Wanderings in the Roman Campagna, London 1908, ed it. a cura di COLOGNOLA, M.: Passeggiate nella Campagna Romana, Roma, 1980, a pág. 267); otros estudios proponen también al ferrarés Jacopo Meleghino entre los técnicos artífices del reajuste urbano de la ciudad; Cfr. PIPITA, G.: La ricostruzione di Frascati, en "Castelli Romani", A. XxXVII (v n. s.), nº. 6, nov. - dic. 1997, págs. 172-173. 

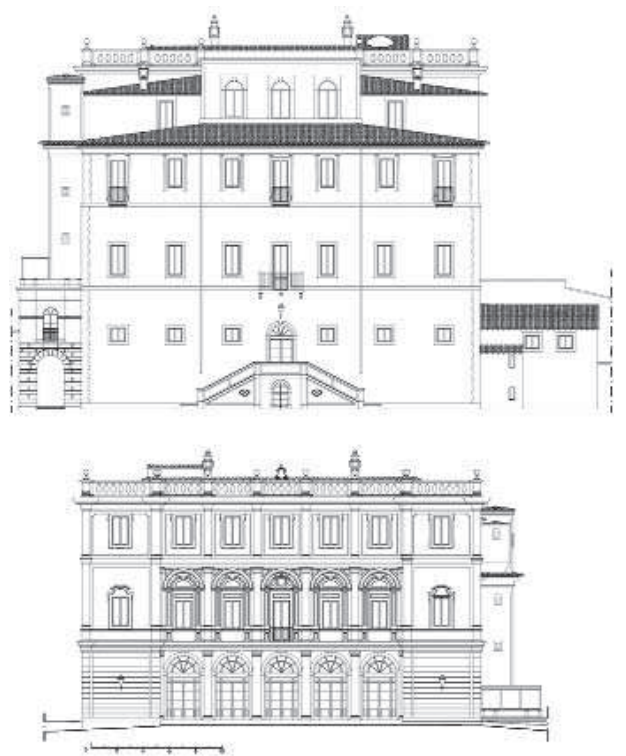

4. Dos ejemplos de fachada.

cincuenta años, todos fueron ocupados (desde luego, donde eran potencialmente idóneos) [4].

- A causa también de la saturación de las posibles áreas donde podíase edificar ex novo, la segunda fase - que corresponde aproximadamente a la primera mitad del siglo XVII - vio una serie de pasajes de propiedad con el incremento, notorio mayormente, de casi todas las estruturas originarias del Complejo. A las Casini se añadieron nuevas alas, según lógicas que correspondían, en su mayor parte, a composiciones simétricas de los volúmenes, requiriendo de su inmediato entorno una disposición igualmente diligente y "digna" de aquellas construcciones, y determinando la edificación de estructuras de carácter arquitectónico-paisajístico de considerable y firme impacto [5].

Es, pues, durante esta fase que la arquitectura de las Villas va definiéndose - en sus aspectos sustanciales - en la forma que, por lo general, se ha mantenido hasta hoy, y también a través de la codificación de algunos temas comunes. De este modo, las fachadas que se orientaban hacia el valle quedaron comprometidas por la disposición - un tanto austera - de las aperturas, mientras amplios porticados y galerías se hallaban situados a fin de articular los frontispicios hacia la altura del Tuscolo 

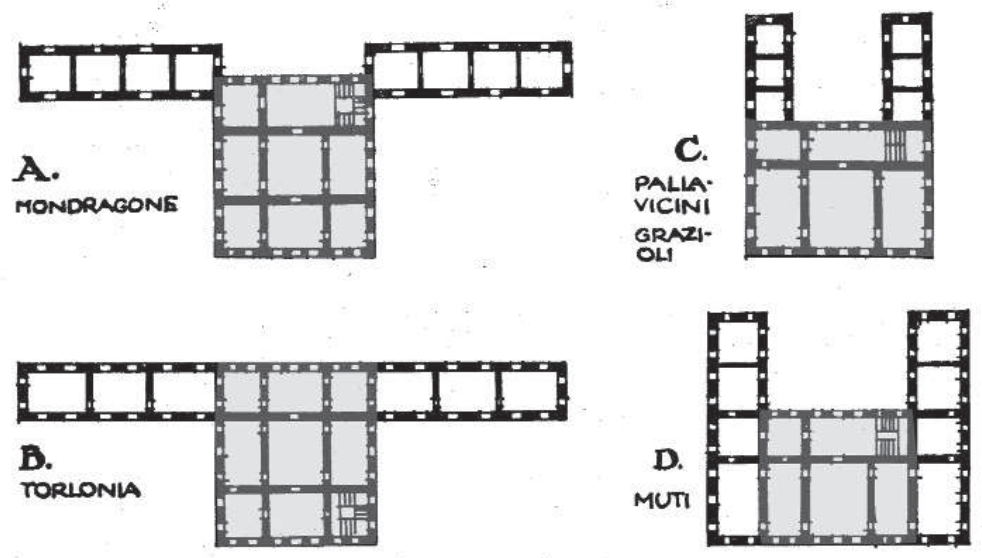

5. Villas: Mondragone, Torlonia, Pallavicini, Grazioli y Muti.

haciéndolos, así, más espaciosos y luminosos. Otras características que se reiteran y tipifican a la casi totalidad de las Villas, y que están en conexión absoluta con las condiciones orográficas locales, se refieren a las soluciones adoptadas para garantizar una verdadera osmosis de las obras con la peculiaridad de las superficies a disposición, situadas, prevalentemente, a media costa.

El problema técnico era resuelto, a menudo, a través de una alegre conjugación de aspectos arquitectónicos, naturales, paisajísticos, escenográficos, estructurales y funcionales. Donde no era posible disponer de un suficiente espacio llano al objeto de levantar las estructuras (adecuado, sobre todo, como divisoria), se proveyó a la creación de murallones de contención que sirvieron también como límites de definición hacia el monte y hacia el valle, de mesetas más o menos amplias. Concretamente, los primeros (al sur) estaban predispuestos para desempeñar funciones de contención del terreno natural, y en varios casos declinados como exposiciones de agua más o menos abundantes y fastuosas los segundos, (al norte), estaban predispuestos para sostener los terraplenes artificiales de las terrazas de 


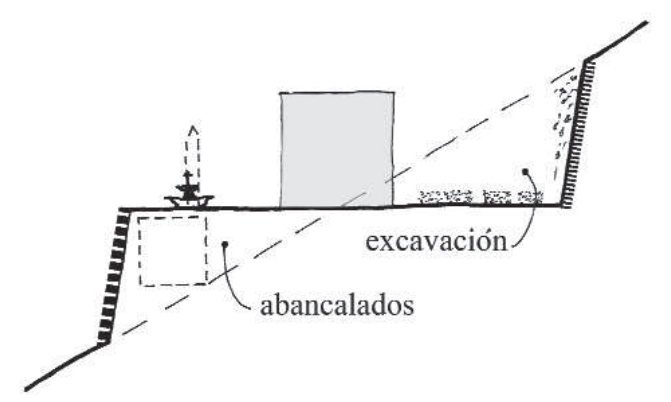

6. Contención del terreno.

expansión (elaboradas fuentes, por lo general, las ornamentaban), participando ambos en la definición de verdaderos podios artificiales de trazado de estribo (en algunos casos incorporaban ambientes de servicio) de los cuales descollaban las majestuosas residencias [6].

Esta doble intervención la encontramos - con ligeras variaciones - en las villas Aldobrandini, Mondragone, Torlonia y Lancellotti (en ésta última, en version reducida en cuanto se refiere a la terraza del valle). Hallamos uno solo de los dos elementos en la Villa Parisi: un pequeño ninfeo a modo de cierre de la corte, en las villas Grazioli y Pallavicini, majestuosas terrazas panorámicas; y en las vilas Rufinella y Falconieri, terrazas de menor resalto [7].

En este contexto de ampliación (aún más que antes) se comprometió al Gotha de los proyectistas que operaban en el área romana ${ }^{14}$, a veces ocupados en el desempeño del rol exclusivo de "arquitecto-fontanero", prepósito a la concepción y ejecución cuidadosa - en algunos casos asociados a expertos "fontaneros" - de complicadas, escenográficas y enrevesadas estructuras hidráulicas ${ }^{15}$. Fue entoces que se llegó a la plena definición del eculiar tejido conectivo entre las Villas: el terri-

14 Para recordar a algunos, podemos citar a Giacomo della Porta, Giovanni Fontana, Giovanni Vasanzio, Carlo Maderno, Flaminio Ponzio y Francesco Borromini.

15 El agua, no tan abundante en Frascati, lo era sí en otras zonas no muy distantes: por eso, el agua de los acueductos prestó apoyo a la construcción de las Villas, pero costaba casi siempre tanto como costaban las construcciones a las que suministraban (con secuelas positivas para la ciudadanía y para el desarrollo agrícola de la zona). Las monumentales y complejas exposiciones de agua se enriquecían a menudo de los ingeniosos juegos y "bromas" para los visitantes, constituyéndose en los siglos siguientes en la delicia de los viajeros, como resulta en algunos grabados y en las descripciones que hace F. Deseine, Rome Moderne, premiere ville de l'Europe, avec toutes ses magnificences et ses delices, Iv, Leide 1713, y C. de Brosses, Lettres familières sur l'Italie / Lettres familières écrites d'Italie en 1739-1740, Paris, 1799. 


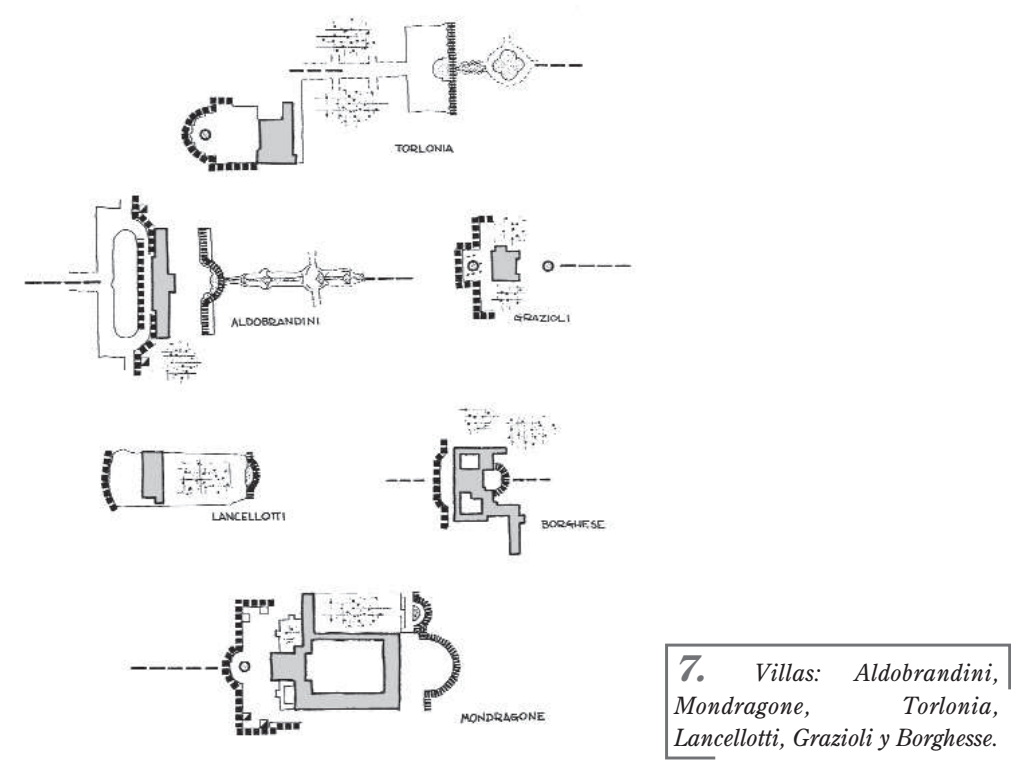

torio no directamente ocupado por los edificios principales y sus anexos fue diseñándose a través de una copiosa trama de pasajes arbolados, ubicados no sólo de manera que pudieran estar conectadas las diversas moradas (entre sí, con los polos religiosos y con la ciudad), constituyendo un sistema puramente funcional a las interrelaciones sociales típicas de la "vida en la villa", sino también acentuando los ejes compositivos de las monumentales obras - llegando a valencias escenográficas de orden paisajístico - y definiendo el amplio contexto ambiental como unicum por su características homogéneas admirablemente captado y representado en la estampa de Matteo Greuter de 162016[8].

- La tercera fase - aunque no haya incidido de modo determinante en la arquitectura de las obras - ha afectado, de cualquier modo, a éstas con transformaciones más tenues, referidas, principalmente, al arreglo externo de jardines y parques, según las cambiantes condiciones del gusto que indujeron las propiedades a modificar las ya seculares estructuras en verde mientras la exigencia de renova-

16 La célebre estampa en tres cuadros Ė fatta celebre la città di Frascati dalla vaghezza delle sue ville suburbane (Kircher en 1671, y Mortier en 1724 la retoman después, sólo por citar las más importantes). 


2: artículos Rodolfo María Strollo

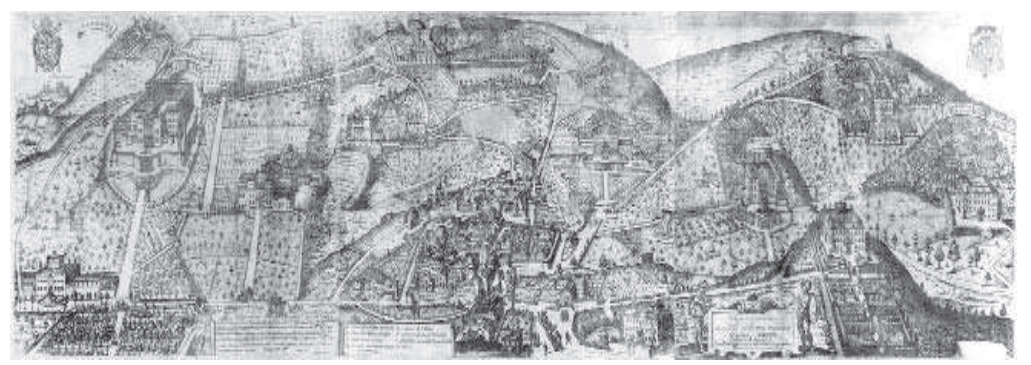

8. Matteo Greuze, 1620 .

ción de los ambientes se manifestó en las limitadas ampliaciones de los edificios (el más ingente tuvo que ver con la Rufinella) o con intervenciones mucho más "epidérmicas" como, por ejemplo, la aparición de nuevos ciclos pictóricos.

Sin embargo, desde este periodo se puede notar un gradual declive del interés y del fasto que habían caracterizado las fases anteriores, debido en gran parte al traslado de la residencia veraniega pontificia a Castel Gandolfo, ocurrido en 1626 por decisión de Urbano VIII (Maffeo Vincenzo Barberini, 1623-1624), cuya motivación la encontramos en la indisponibilidad, en Frascati, de un lugar apropiado para la edificación de una estructura "digna" del linaje del nuevo Pontífice. Así, pues, el interés del entourage papal por el Área Tuscolana empezó a mermar, y en muchas majestuosas residencias diose inicio al lento proceso de transformación funcional, el cual se contó entre las primeras causas de la alteración de las estructuras originarias y, contemporáneamente, del territorio que las enlazaba ${ }^{17}$.

- A excepción de las villas Aldobrandini y Lancellotti - aún pertenecientes a las respectivas familias - las demás residencias nobiliarias pasaron de una mano a otra generalmente en torno al año 1900, y después, por largo tiempo, resultaron siendo de propiedad de los mismos compradores.

Los intensos bombardeos de los Aliados que a partir de septiembre de 194318 recayeron sobre Frascati, resultaron fatales para las villas Pallavicini, Torlonia y Vecchia pues terminaron, prácticamente, por el ras del suelo; después de removidos los escombros se hicieron construcciones modernas (en el caso de las dos últimas

17 La relevante intervención transformadora de Vanvitelli (por encargo de los Jesuitas que la habían comprado en 1740) pone a la entidad edil "fuera del cánone" con respecto a las demás, convirtiéndola en una estructura de tipo casa-convento acorde con las exigencias de la Orden. 
villas, alardeando una presunta fidelidad a las formas de las antiguas construcciones). La suerte de las demás estructuras, si no se decidió por daños directos como sucedió con las tres ya citadas (la villa Aldobrandini también quedó dañada seriamente, y aún más la Villa Falconieri pues perdió el ala derecha), se decidió por otros trágicos acontecimientos ligados a los eventos bélicos, como por ejemplo: la ocupación de las tropas y, sobre todo, a los evacuados que, en vasto número se refugiaron allí.

Algunas Villas, no obstante, habían sido ya objeto (o de cualquier modo lo fueron tras el conflicto bélico) de relevantes transformaciones a fin de adecuarlas a las nuevas funciones, por cierto, desiguales a las originarias. específicamente, esto fue válido (limitado sólo a las estructuras ediles) para las villas Mondragone, Rufinella y Sora. La magnitud de las intervenciones fue de tal impacto global que hizo lícito individuar, en este periodo, una verdadera "cuarta fase", definible como la de las "demudaciones utilitaristas"19.

Las modificaciones que se efectuaron fueron tan desastrosas ( $y$, mayormente, irreversibles) que casi era preferible la degradación de algunas obras, como las villas Grazioli, Lancellotti y Muti continuaban manifestando antes de ser objeto, recientemente, de radicales y discutibles trabajos de restauración.

Se recuerdan, en seguida, los ulteriores daños perpetrados, desde finales de la segunda conflagración mundial hasta nuestros días, de jardines y, más todavía de parques: éstos se han convertido en ámbitos apetecibles de intervención edil, entre los que se cuentan no sólo los múltiples asaltos de la especulación edil, sino también algunas consistentes $\mathrm{y}$ «desconsideradas» intervenciones del sector público. ${ }^{20}$

18 A causa de la presencia - en la zona - del alto mando alemán en el frente sur (OBS OberBefershaberSüd), el área sufrió numerosos ataques aéreos: inicialmente - de modo intenso - el 8 de septiembre de 1943 y sucesivamente con repetidas incursiones hasta junio de 1944. Los datos oficiales sobre los efectos del bombardeo señalan que el $76,5 \%$ de las construcciones de la ciudad resultaron total o parcialmente destruidas, el $23 \%$ dañadas y el $87 \%$ de la población quedó sin techo.

19 Cfr. Strollo, R.M.: II Complesso delle..., Op. Cit.

20 Los estratos de algunas Villas han sufrido con el tiempo la corrosión de la erosión edil, de modo más o menos moderado (tal como les ha ocurrido, por ejemplo, a las villas Grazioli y Torlonia); otras (verbigracia, la Villa Pallavicini) se han encuadrado totalmente en la edificación. Sobresalen, asimismo, con singular evidencia, las múltiples acciones en perjuicio del Status Borghesianum, ocurridas con la venia de dos Administraciones pertinentes (o, incluso, tras sus "motivaciones", como la insensata planificación urbanística que el Ayuntamiento de Monte Porzio Catone demuestra). Éstas han erosionado buena parte del estrato con intervenciones violentas, tanto privadas como públicas. Entre las primeras, una de las más recientes y devastadoras ha tenido que ver con las construcciones del Barco del siglo XVI, transformadas en una residencia "exclusiva" de discutible tonalidad "rústico-elegante"; entre las segundas, recordamos el asentamiento de casas económicas en el parterre del Palazzetto Mergè y la construcción de grandes edificios como el de ENPAS (Ente Nacional de Seguridad Social Asistencia yo el de ENPAS (Ente Nacional de Seguridad Social y Asistencia dependientes del Estado) al reparo de la Villa Parisi. Siempre en cuanto respecta a las intervenciones públicas dentro del Complejo de las Villas, no se olvidan las numerosas alteraciones de las áreas verdes, lo cual daba lugar a su propia "liquidación" (limitándonos las ya en acto tenemos, por ejemplo, la calle que corta el fondo de la Villa Aldobrandini a la ciudad, o, el centro deportivo - con piscinas - contiguo al parque de la Villa Torlonia, que, por lo demás, se encuentra abandonado y en total degradación. 
Todo ello en concomitancia con el sustancial desinterés de la Administración Pública comprometida con los aspectos de mayor relieve del patrimonio que constituyen las Villas Tuscolanas, ha contribuido - a pesar de la multiplicidad de iniciativas promocionales de escaso carácter turístico-comercial - a dejar aparte los auténticos valores del Complejo, y a mellar gravosamente justo aquel aspecto de homogeneidad paisajística que constituía su principal peculiaridad21.

21 Además de Frascati, los Ayuntamientos de Monte Porzio Catone y de Grottaferrata se dividen la jurisdicción administrativa del grupo, situación que, a menudo, ha causado incoherencia y discontinuidad de las intervenciones, e insuficiencia de tutela también, pues las Administraciones locales parecen haber considerado el territorio, casi sempre, y, sobre todo, como un recurso económico a explotar, que, vinculado al constantemente activo saldo migratorio (comprendido, en buena parte, por los núcleos familiares que se transfieren del áre urbana de Roma en búsqueda de un ambiente agreste transfieren del área urbana de Roma en búsqueda de un ambiente agreste o de aire puro, que, hoy por hoy, resultan ser sólo un recuerdo. Tampoco la situación ha mejorado con la entrada en acción del IRViT (Istituto Regional para las Villas Tuscolanas, instituido por Ley Regional núm. 43 del 6.XI.1992) que, en teoría, también debería ser un preciso propósito a fines de tutela y valorización culturalmente cualificados. Téngase presente, a modo de ejemplo, que el Comité Técnico Científico sancionado por el Estatuto del Ente - del cual quien escribe esto es, en el papel, miembro desde 2004 - no ha sido convocado nunca desde su institución, por ninguna de las dos mayorías políticas opuestas que han administrado la Región Lacio (con los consiguientes cambios en la Presidencia y en el organigrama del Consejo Directivo del Ente mismo). 